\title{
A Leslie-Gower Holling Type-II Predator-Prey Mathematical Model with Disease in Prey Population Incorporating a Prey Refuge
}

\author{
P. Mandal, N. Das and S. Pal \\ 1. Department of Mathematics, Hooghly Mohsin College, Chinsurah, Hooghly, India. \\ 2. Department of Mathematics, Maulana Azad College, Kolkata, India. \\ 3. Department of Mathematics, University of Kalyani, Nadia, India.
}

Received: July 25, 2016 / Accepted: August 23, 2016 / Published: October 25, 2016.

\begin{abstract}
We formulate and analyze a predator-prey model followed by Leslie-Gower model in which the prey population is infected by disease. We assume that the disease can only spread over prey population. As a result prey population has been classified into two categories, namely susceptible prey, infected prey where as the predator population remains free from infection. To investigate the behaviour of prey population we incorporate prey refuge in this model. Since the prey refuge decreases the predation rate then it has an important effect in our predator-prey interaction model. We have discussed the existence of various equilibrium points and local stability analysis at those equilibrium points. We investigate the Hopf-bifurcation analysis about the interior equilibrium point by taking the rate of infection parameter and the prey refuge parameter as bifurcation parameters. The numerical analysis is carried out to support the analytical results and to discuss some interesting results that our model exhibits.
\end{abstract}

Keywords: Predator and prey, Disease transmission, Prey refuge, Stability, Hopf-bifurcation.

\section{Introduction}

In theoretical ecology the dynamical relationship between prey and predator is one of the important themes from the point of view of its existence. Also mathematical modelling is essential to understand the dynamical behaviour of such system. Anderson and May [1], Hadeler and Freedman [2] analyzed eco-epidemic models where predator population is infected through eating prey. The mathematical models, like $[3,4,5,6,7]$ on prey-predator system have been studied by the researchers, out of which the interesting dynamics of Holling Tanner model [7] plays an important role in the theoretical ecology. Leslie $[8,9]$ introduced prey-predator model in which the carrying capacity of the predators environment is proportional to the number of prey. Incorporating the

Corresponding author: S. Pal, Department of Mathematics, University of Kalyani, Nadia, India.
Holling type-II functional response the modified Leslie Gower prey-predator model was obtained, for example [10]. On the other hand spatial prey refuge is one of the more relevant behavioral traits that affect the dynamics of predator-prey systems. Some of the researchers $[6,10,11,12,13]$ have investigated the influence of prey refuge and they concluded that the refuge used by the prey has a stabilizing effect on the prey-predator interaction. In epidemiology, the incidence rate in mathematical models of infectious disease $[14,15]$ play an important role. In [16] the authors analyzed an eco-epidemic model incorporating a prey refuge in predator-prey system in which we observe the very interesting dynamics corresponding to refuge term. Recently, the effect of disease on ecological systems is an important issue from mathematical as well as experimental point of view as the effect of infectious disease on the ecological 
system controls the size of the populations. Venturino [17, 18], Haque and Venturino [19], Haque et al. [20, 21, 22], Xiao and Chen [23, 24], Tewa [25], Hethcote [26], Rahman [27], Chattopadhyay et.all [28,29,30] discussed the dynamics of preypredator system with disease in prey population.

In this paper, we have considered a modified Leslie-Gower and Holling type-II predator-prey model. In this model we include the infectious disease in prey population as well as prey refuge term. Incorporation of infected prey refuge leaves a factor of the infected prey species which are accessible to the predator. This model becomes more realistic than the existing models in ecological as well as epidemiological point of view after incorporation of such effect. The basic assumptions and model formulation is discussed in Section 2. In Section 3, we have discussed the boundedness of the solutions in the positive region. Section 4 deals with the equilibrium points, their existence and stability analysis. Also the influence of infected prey refuge on each population and Hopf -bifurcation analysis at interior equilibrium point is analyzed. All our important findings are numerically verified in Section5. Finally, Section 6 contains the discussion and biological implications of our mathematical findings.

\section{The Basic Assumptions and Model Formulation}

After the brief introduction we formulate the mathematical model which is based on the following assumptions:

(1) At any time $t(>0)$ the total prey population is denoted by $n$ and the total predator population is denoted by $z$.

(2) In the presence of disease total prey population $n$ is divided into susceptible prey $x$ and infected prey $y$, so that the total prey population is $n=x+y$.

(3) We assume that the disease can only transmitted from susceptible prey $x$ to infected prey $y$ but it does not spread from any kinds prey to predator by feeding or any other ways.

(4) We assume that the susceptible prey is capable of reproducing according to the logistic law $\frac{d x}{d t}=r x\left(1-\frac{x+y}{k}\right) \quad$ with carrying capacity $k$ and intrinsic birth rate $r$.

(5) The susceptible prey $x$ becomes infected following the mass action law at constant rate of infection $\alpha$.

(6) Predators consume susceptible prey and infected prey following Holling type-II functional response at constant rate $c_{1}, c_{2}$ respectively.

(7) $m y$ is the capacity of prey refuge at time $t$ and so refuge protecting $m y$ of the infected prey, where $0<m<1$. This leaves $(1-m) y$ of the infected prey available to the predator.

On the basis of the above assumptions we the following mathematical model:

$$
\begin{gathered}
\frac{d x}{d t}=r x\left(1-\frac{x+y}{k}\right)-\alpha x y-c_{1} \frac{x z}{\left(x+(1-m) y+k_{1}\right)}, \\
\frac{d y}{d t}=\alpha x y-c_{2} \frac{(1-m) y z}{\left(x+(1-m) y+k_{2}\right)}-d y, \quad(1) \\
\frac{d z}{d t}=\left(a-c_{3} \frac{z}{\left(x+(1-m) y+k_{2}\right)}\right) z,
\end{gathered}
$$

where $a$ is the growth rate of predator, $c_{3}$ is the maximum value of per-capita reduction rate of the predator, $k_{1}, k_{2}$ are the half saturation constants for prey and predator population respectively and $d$ is the death rate of infected prey.

The system (1) has to be analyzed with the following initial conditions,

$$
x(0)>0, y(0)>0, z(0)>0 .
$$

\section{Qualitative Analysis of the System}

\subsection{Boundedness of the System}

Theorem 1. All the solutions of the system (1) are bounded.

Proof: Consider the function $u(t)=x(t)+y(t)+z(t)$. Now using the equations 
(1), we have

$$
\begin{gathered}
\frac{d u}{d t}=\frac{d x}{d t}+\frac{d y}{d t}+\frac{d z}{d t}= \\
r x\left(1-\frac{x+y}{k}\right)-c_{1} \frac{x z}{x+(1-m) y+k_{1}}-d y-c_{2} \frac{(1-m) y z}{x+(1-m) y+k_{2}} \\
+\left(a-c_{3} \frac{z}{x+(1-m) y+k_{2}}\right) z \\
\leq r x-\frac{r}{k} x^{2}-\frac{r}{k} x y-d y+\left(a-c_{3} \frac{z}{x+(1-m) y+k_{2}}\right) z .
\end{gathered}
$$

Therefore

$$
\begin{gathered}
\frac{d u}{d t}+\mu u \leq \\
x\left(r+\mu-\frac{r x}{k}\right)-(d-\mu) y+\left\{a+\mu-\frac{c_{3} z}{x+(1-m) y+k_{2}}\right\} z \\
\leq x\left(r+\mu-\frac{r x}{k}\right)-(d-\mu) y+\left\{\frac{x+(1-m) y+k_{2}}{4 c_{3}}\right\}(a+\mu)^{2} \\
=x\left\{r+\mu-\frac{r x}{k}+\frac{(a+\mu)^{2}}{4 c_{3}}\right\}-\left\{d-\mu-\frac{(1-m)(a+\mu)^{2}}{4 c_{3}}\right\} y \\
+\frac{k_{2}(a+\mu)^{2}}{4 c_{3}} \leq \frac{k}{4 r}\left\{r+\mu+\frac{(a+\mu)^{2}}{4 c_{3}}\right\}+\frac{k_{2}(a+\mu)^{2}}{4 c_{3}}
\end{gathered}
$$

Now if $d>\frac{(1-m) a^{2}}{4 c_{3}}$, we can choose $\mu$ in such a way that $d>\mu+\frac{(1-m)(a+\mu)^{2}}{4 c_{3}}$ then the right hand side of the above inequality is bounded. Then we can find a constant $P>0$, such that $\frac{d u}{d t}+m u<P$.

Now by the theory of differential inequality ( Birkhoff and Rota [31]) we have,

$$
0 \leq u(t) \leq \frac{P}{\mu}\left(1-e^{-\mu t}\right)+u(0) e^{-\mu t} .
$$

As $t \rightarrow \infty$, then $0 \leq u(t) \leq \frac{P}{\mu}$. Hence $u(t)$ is a bounded quantity.

Thus all the solutions of the system (1) are confined in the region

$\Omega=\left\{(x, y, z): 0 \leq x(t)+y(t)+z(t) \leq \frac{P}{\mu}+\varepsilon\right.$, for all $\left.\varepsilon>0\right\}$.

\subsection{Equilibria Analysis}

- The equilibria $\mathbf{E}_{0}(0,0,0)$ and $\mathbf{E}_{1}(k, 0,0)$ exist for all parametric values.
- The prey free equilibrium point $\mathbf{E}_{2}\left(0,0, \frac{a k_{2}}{c_{3}}\right)$ exists for all parametric values.

- The predator free equilibrium point $\mathbf{E}_{3}\left(\frac{d}{\alpha}, \frac{r(k \alpha-d)}{\alpha(k \alpha+r)}, 0\right)$ exists if $R_{1}=\frac{k \alpha}{d}>1$.

- The infected prey free equilibrium point $\mathbf{E}_{4}(\bar{x}, 0, \bar{z})$ exists if $R_{2}=\frac{k_{1} r c_{3}}{k_{2} a c_{1}}>1$, where $\bar{z}=\frac{a}{c_{3}}\left(\bar{x}+k_{2}\right)$ and $\bar{x}$ is the positive root of the equation $A x^{2}+B x-C=0$ and the coefficients are given by

$$
\begin{aligned}
& \begin{array}{l}
A=c_{3} r, \\
B
\end{array}=\left(k a c_{1}+k_{1} c_{3} r-k r c_{3}\right), \\
& C=k\left(k_{1} r c_{3}-k_{2} a c_{1}\right) \\
& \text { - The positive interior equilibrium point } \\
& \mathbf{E}^{*}\left(x^{*}, y^{*}, z^{*}\right), \\
& \text { where } \\
& \qquad x^{*}=\frac{1}{\alpha}\left\{d+\frac{a c_{2}(1-m)}{c_{3}}\right\}=p,(\text { say }), \\
& \quad y^{*}=\frac{1}{a(1-m)}\left(c_{3} z^{*}-a x^{*}-a k_{2}\right)
\end{aligned}
$$

where

and $z^{*}$ is the positive root of the equation $A z^{2}-B z-C=0$ and the coefficients are given by

$$
\begin{aligned}
& A=c_{3}^{2}(r+k \alpha), \\
& B=a(1-m)\left\{c_{3} r(k-p)-k a c_{1}\right\} \\
& +a c_{3}(r+k \alpha)\left(2 k_{2}-k_{1}+p\right), \\
& C=a^{2}\left(k_{1}-k_{2}\right)\left\{\begin{array}{l}
r(1-m)(k-p) \\
+\left(k_{2}+p\right)(r+k \alpha)
\end{array}\right\} \text {. }
\end{aligned}
$$

\subsection{Stability Analysis}

The stability of the system (1) at each equilibria is obtained by using Routh-Hurwitz stability criterion.

For this we have to compute the variational matrix $V(x, y, z)$ of the system (1) and check the stability at each equilibrium.

Result 1. The equilibrium point $\mathbf{E}_{0}:(0,0,0)$ is unstable as one eigenvalue of the variational matrix is 
positive.

Result 2. The equilibrium point $\mathbf{E}_{1}:(k, 0,0)$ is unstable as one eigenvalue of the variational matrix is positive.

Result 3. The susceptible prey and infected prey free equilibrium point $\mathbf{E}_{2}\left(0,0, \frac{a k_{2}}{c_{3}}\right)$ is stable if $R_{01}=\frac{r c_{3} k_{1}}{a c_{1} k_{2}}<1$

Result 4. The predator free equilibrium point $\mathbf{E}_{3}\left(\frac{d}{\alpha}, \frac{r(k \alpha-d)}{\alpha(k \alpha+r)}, 0\right)$ is unstable as one eigenvalue of the variational matrix is positive.

Result 5. The infected prey free equilibrium point $\mathbf{E}_{4}(\bar{x}, 0, \bar{z}) \quad$ is stable if $R_{02}<1$, where $R_{02}=\frac{\alpha c_{3} \bar{x}}{\left(a c_{2}+d c_{3}\right)}$.(For proof see Appendix.)

Stability analysis of the positive interior equilibrium and Hopf bifurcation

Theorem 2. The positive interior Equilibrium point $E^{*}\left(x^{*}, y^{*}, z^{*}\right)$ is locally asymptotically stable if the following conditions hold $d_{1}>0$, $d_{3}>0, d_{1} d_{2}-d_{3}>0$, where $d^{\prime}{ }_{i} s$ are given in the proof of the theorem.

Proof: The variational matrix of the system (1) around the positive equilibrium point $E^{*}\left(x^{*}, y^{*}, z^{*}\right)$ is

$$
V^{*}=\left[\begin{array}{ccc}
-m_{11} & -m_{12} & -m_{13} \\
m_{21} & m_{22} & -m_{23} \\
m_{31} & m_{32} & -m_{33}
\end{array}\right]
$$

where

$$
\begin{aligned}
& m_{11}=\left\{\frac{r}{k} x^{*}-\frac{c_{1} * x * z^{*}}{\left[x^{*}+(1-m) y^{*}+k_{1}\right]^{2}}\right\}>0, \\
& m_{12}=\left(\frac{r}{k}+\alpha\right) x^{*}-\frac{c_{1}(1-m) x^{*} z^{*}}{\left[x^{*}+(1-m) y^{*}+k_{1}\right]^{2}}>0, \\
& m_{13}=\frac{c_{1} x^{*}}{x^{*}+(1-m) y^{*}+k_{1}}>0 ; \\
& m_{21}=\alpha y^{*}+\frac{c_{2}(1-m) y^{*} z^{*}}{\left[x^{*}+(1-m) y^{*}+k_{2}\right]^{2}}>0, \\
& m_{22}=\alpha x^{*}-d-\frac{c_{2}\left(x^{*}+k_{2}\right) z^{*}}{\left[x^{*}+(1-m) y^{*}+k_{2}\right]^{2}}>0, \\
& m_{23}=\frac{c_{2}(1-m) y^{*}}{x^{*}+(1-m) y^{*}+k_{2}}>0 ;
\end{aligned}
$$

$$
m_{31}=\frac{a^{2}}{c_{3}}>0, m_{32}=\frac{a^{2}(1-m)}{c_{3}}>0, m_{33}=a>0 .
$$

It is found that the characteristic roots of the variational matrix $V^{*}$ at $E^{*}$ are the roots of the equation $\rho^{3}+d_{1} \rho^{2}+d_{2} \rho+d_{3}=0$, where

$$
\begin{aligned}
& d_{1}=\left(m_{11}-m_{22}+m_{33}\right), \\
& d_{2}=m_{33}\left(m_{11}-m_{22}\right)+m_{13} m_{31} \\
& +m_{12} m_{21}+m_{23} m_{32}-m_{22} m_{11} \text {, } \\
& d_{3}=m_{12}\left(m_{21} m_{33}-m_{31} m_{23}\right) \\
& +m_{13}\left(m_{21} m_{32}-m_{31} m_{22}\right) \\
& +m_{11}\left(m_{23} m_{32}-m_{22} m_{33}\right) \text {, } \\
& d_{1} d_{2}-d_{3}=\left(m_{11}-m_{22}\right)\left\{\begin{array}{l}
m_{12} m_{21} \\
+m_{11}\left(m_{33}-m_{22}\right)
\end{array}\right\} \\
& +\left(m_{33}-m_{22}\right)\left\{m_{23} m_{32}+m_{33}\left(m_{11}-m_{22}\right)\right\} \\
& +m_{13}\left(m_{11} m_{31}-m_{33} m_{32}\right) \\
& +m_{32}\left(m_{12} m_{23}-m_{21} m_{13}\right) \text {. }
\end{aligned}
$$

By the Routh-Hurwitz criteria, all roots of the above characteristic equation have negative real parts if and only if $d_{1}>0, d_{3}>0, d_{1} d_{2}-d_{3}>0$. Therefore, the positive interior equilibrium point $E^{*}\left(x^{*}, y^{*}, z^{*}\right)$ is locally asymptotically stable if $d_{1}>0, d_{3}>0, d_{1} d_{2}-d_{3}>0$.

\subsection{Influence of infected prey refuge}

In this part, we shall analyze the influence of infected prey refuge (i.e., the effect of refuge parameter $m$ ) on each population densities when the interior equilibrium point $E^{*}\left(x^{*}, y^{*}, z^{*}\right)$ of system (1) exists and is stable.

We assume that the following system of equations for the system (1) without infected prey refuge.

$$
\begin{gathered}
\frac{d x}{d t}=r x\left(1-\frac{x+y}{k}\right)-\alpha x y-c_{1} \frac{x z}{\left(x+y+k_{1}\right)}, \\
\frac{d y}{d t}=\alpha x y-c_{2} \frac{y z}{\left(x+y+k_{2}\right)}-d y, \\
\frac{d z}{d t}=\left(a-c_{3} \frac{z}{\left(x+y+k_{2}\right)}\right) z
\end{gathered}
$$


Let $\left(\bar{x}^{*}, \bar{y}^{*}, \bar{z}^{*}\right)$ be the equilibrium point for the system (3), where

$$
\begin{aligned}
& \bar{x}^{*}=\frac{1}{\alpha}\left\{d+\frac{a c_{2}}{c_{3}}\right\}=p^{*},(\text { say }), \\
& \bar{y}^{*}=\frac{1}{a}\left(c_{3} \bar{z}^{*}-a \bar{x}^{*}-a k_{2}\right)
\end{aligned}
$$

and $\bar{z}^{*}$ is the positive root of the equation $A z^{2}-B z-C=0$ and the coefficients are given by

$$
\begin{aligned}
& A=c_{3}^{2}(r+k \alpha), \\
& B=a\left\{c_{3} r\left(k-p^{*}\right)-k a c_{1}\right\} \\
& +a c_{3}(r+k \alpha)\left(2 k_{2}-k_{1}+p^{*}\right), \\
& C=a^{2}\left(k_{1}-k_{2}\right)\left\{\begin{array}{l}
r\left(k-p^{*}\right) \\
+\left(k_{2}+p^{*}\right)(r+k \alpha)
\end{array}\right\} .
\end{aligned}
$$

(i) Influence of infected prey refuge on susceptible prey population:

Clearly,

$$
\frac{1}{\alpha}\left\{d+\frac{a c_{2}(1-m)}{c_{3}}\right\}<\frac{1}{\alpha}\left\{d+\frac{a c_{2}}{c_{3}}\right\}, \forall m \in(0,1)
$$

This inequality shows that, for any fixed $m \in(0,1)$, the infected prey refuge can decrease susceptible prey density.

As $x^{*}$ is a continuous function of parameter $m$,we have

$$
\frac{d x^{*}}{d m}=-\frac{a c_{2}}{\alpha c_{3}}<0, \forall m \in(0,1) .
$$

Thus, $x^{*}$ is a strictly decreasing function of $m$, i.e., increasing the amount of infected prey refuge can decrease susceptible prey density when the equilibrium $E^{*}\left(x^{*}, y^{*}, z^{*}\right)$ of system (1) is stable.

(ii) Influence of infected prey refuge on predator population:

Now,

$$
\begin{aligned}
z^{*} & =\frac{B+\sqrt{\left(B^{2}+4 A C\right)}}{2 A}, \\
\bar{z}^{*} & =\frac{B^{\prime}+\sqrt{\left(B^{\prime 2}+4 A C^{\prime}\right)}}{2 A}, \text { where } \\
A & =c_{3} c_{3}(r+k \alpha), \\
B & =a(1-m)\left\{c_{3} r(k-p)-k a c_{1}\right\}+a c_{3}(r+k \alpha)\left(2 k_{2}-k_{1}+p\right) \\
& =a(1-m)\left(c_{3} r k-k c_{1} a\right)+c_{3} r a p m+a c_{3}(r+k \alpha)\left(2 k_{2}-k_{1}\right)+p a c_{3} k \alpha, \\
C & =a^{2}\left(k_{1}-k_{2}\right)\left\{r(1-m)(k-p)+\left(k_{2}+p\right)(r+k \alpha)\right\} \\
& =a^{2}\left(k_{1}-k_{2}\right)\left(r k+m r p+k_{2} r+k_{2} k \alpha+p k \alpha-m r k\right), \\
B^{\prime} & =a\left(c_{3} r k-k c_{1} a\right)+a c_{3}(r+k \alpha)\left(2 k_{2}-k_{1}\right)+p^{*} a c_{3} k \alpha, \\
C^{\prime} & =a^{2}\left(k_{1}-k_{2}\right)\left(r k+k_{2} r+k_{2} k \alpha+p^{*} k \alpha\right),
\end{aligned}
$$

Clearly, $B<B^{\prime}$ and $C<C^{\prime}, \forall m \in(0,1)$ then $\frac{B+\sqrt{\left(B^{2}+4 A C\right)}}{2 A}<\frac{B^{\prime}+\sqrt{\left(B^{\prime 2}+4 A C^{\prime}\right)}}{2 A}$ i.e., $z^{*}<\bar{z}^{*}$

This inequality shows that, for any fixed $m \in(0,1)$, the infected prey refuge can decrease predator density.

Now, $z^{*}$ is a continuous function of parameter $m$, we have
Since,

$$
\begin{aligned}
\frac{d B}{d m} & =-a\left\{\begin{array}{l}
c_{3} r(k-p)-k a c_{1} \\
+a c_{2}(k \alpha+r m)
\end{array}\right\}<0, \\
\frac{d C}{d m} & =-a^{2}\left(k_{1}-k_{2}\right)\left(r k+\frac{a m r c_{2}}{\alpha c_{3}}\right. \\
+ & \left.\frac{a k c_{2} \alpha}{c_{3}}+r k-p r\right)<0,
\end{aligned}
$$


Therefore,

$$
\frac{d z^{*}}{d m}=\left(z^{*} \frac{d B}{d m}+\frac{d C}{d m}\right) \frac{1}{\sqrt{\left(B^{2}+4 A C\right)}}<0,
$$

Thus, $z^{*}$ is a strictly decreasing function of $m$, i.e., increasing the amount of infected prey refuge can decrease predator density when the equilibrium $E^{*}\left(x^{*}, y^{*}, z^{*}\right)$ of system (1) is stable.

(iii) Influence of infected prey refuge on infected prey population:

Obviously, $y^{*}=\frac{1}{a(1-m)}\left(c_{3} z^{*}-a x^{*}-a k_{2}\right)>$

$$
\frac{1}{a}\left(c_{3} \bar{z}^{*}-a \bar{x}^{*}-a k_{2}\right)=\bar{y}^{*} \forall m \in(0,1)
$$

Thus for any fixed $m \in(0,1)$, the infected prey refuge can increase infected prey density, it is expected.

Since $y^{*}$ is a continuous function of parameter $m$, we have

$\frac{d y^{*}}{d m}=\frac{c_{3}}{a(1-m)} \frac{d z^{*}}{d m}+\frac{c_{3} z^{*}}{a(1-m)^{2}}+$

$\frac{a c_{2}}{\alpha c_{3}(1-m)}-\frac{x^{*}}{(1-m)^{2}}-\frac{\alpha k_{2}}{(1-m)^{2}}>0, \forall m \in(0,1)$.

Therefore, $y^{*}$ is a strictly increasing function of $m$, i.e., increasing the amount of infected prey refuge can increase the infected prey density when the interior equilibrium point $E^{*}\left(x^{*}, y^{*}, z^{*}\right)$ of system (1) is stable.

Theorem 3. The Hopf-bifurcation of the equivalent equilibrium $E^{*}$ occurs at $\lambda=\lambda^{*} \in(0, \infty)$ if and only if

(i) $\Psi\left(\lambda^{*}\right)=d_{1}\left(\lambda^{*}\right) d_{2}\left(\lambda^{*}\right)-d_{3}\left(\lambda^{*}\right)=0$,

(ii) $\left[\frac{d \operatorname{Re}\left(\rho_{j}(\lambda)\right)}{d \lambda}\right]_{\lambda=\lambda^{*}} \neq 0$

where $\rho(\lambda)$ is purely imaginary at $\lambda=\lambda^{*}$.

Proof: By the condition $\Psi\left(\lambda^{*}\right)=0$, the characteristic equation ca be written as

$$
\left(\rho^{2}+d_{2}\right)\left(\rho+d_{1}\right)=0 .
$$

If this equation has three roots, say $\rho_{1}=+i \sqrt{d_{2}}, \rho_{2}=-i \sqrt{d_{2}}, \rho_{3}=-d_{1}$. For all $\lambda$, the

roots are in general form

$$
\begin{gathered}
\rho_{1}(\lambda)=\beta_{1}(\lambda)+i \beta_{2}(\lambda), \\
\rho_{2}(\lambda)=\beta_{1}(\lambda)-i \beta_{2}(\lambda), \\
\rho_{3}(\lambda)=-d_{1}
\end{gathered}
$$

Now, we shall verify the transversally condition

$$
\left[\frac{d \operatorname{Re}\left(\rho_{j}(\lambda)\right)}{d \lambda}\right]_{\lambda=\lambda^{*}} \neq 0, j=1,2
$$

Substituting

$$
\rho_{j}(\lambda)=\beta_{1}^{\prime}(\lambda)+i \beta_{2}(\lambda)
$$

into (4) and calculating the derivative, we have

$$
\begin{gathered}
K(\lambda) \beta^{\prime}{ }_{1}(\lambda)-L(\lambda) \beta^{\prime}{ }_{2}(\lambda)+M(\lambda)=0, \\
L(\lambda) \beta^{\prime}{ }_{1}(\lambda)+K(\lambda) \beta^{\prime}{ }_{2}(\lambda)+R(\lambda)=0
\end{gathered}
$$

where

$$
\begin{aligned}
& K(\lambda)=3 \beta_{1}^{2}(\lambda)+2 d_{1}(\lambda) \beta_{1}(\lambda)+d_{2}(\lambda)-3 \beta_{2}^{2}(\lambda) \\
& \begin{aligned}
L(\lambda) & =6 \beta_{1}(\lambda) \beta_{2}(\lambda)+2 d_{1}(\lambda) \beta_{2}(\lambda) \\
M(\lambda) & =\beta_{1}^{2}(\lambda) d^{\prime}{ }_{1}(\lambda)+d^{\prime}{ }_{2}(\lambda) \beta_{1}(\lambda) \\
& +d^{\prime}{ }_{3}(\lambda)-d^{\prime}{ }_{1}(\lambda) \beta_{2}^{2}(\lambda)
\end{aligned} \\
& R(\lambda)=2 \beta_{1}(\lambda) \beta_{2}(\lambda) d^{\prime}{ }_{1}(\lambda)+d^{\prime}{ }_{2}(\lambda) \beta_{2}(\lambda) .
\end{aligned}
$$

Since

$$
L\left(\lambda^{*}\right) R\left(\lambda^{*}\right)+K\left(\lambda^{*}\right) M\left(\lambda^{*}\right) \neq 0
$$

We have

$$
\left[\frac{d \operatorname{Re}\left(\rho_{j}(\lambda)\right)}{d \lambda}\right]_{\lambda=\lambda^{*}}=-\left.\frac{L R+K M}{K^{2}+L^{2}}\right|_{\lambda=\lambda^{*}} \neq 0
$$

and

$$
\rho_{3}\left(\lambda^{*}\right)=-d_{1}\left(\lambda^{*}\right) \neq 0 .
$$

Therefore the transversally condition holds. This 
implies that a Hopf-bifurcation occurs at $\lambda=\lambda^{*}$ and is non-degenerate. This complete the proof.

\section{Numerical Simulations}

In this section, our study focused on the occurrence and termination of the disease. We begin with a parameter set (see Table1) for which the existence conditions of the coexistence equilibrium point $E^{*}$ are satisfied and the coexistence equilibrium point $E^{*}=(16.3269,24.1516,23.8171)$ is locally asymptotically stable in the form of a stable focus with eigenvalues $-0.03106 \pm i 2.5349,-0.3365$
(cf.Fig1). Keeping the other parameter fixed and increasing the value of the parameters $m=0.95$, we observe that the solution of (1) changes from stable behavior to oscillatory behavior (cf.Fig 2). Also, keeping the other parameter fixed and increasing the value of the parameters $\alpha=0.12$, we observe that the solution of (1) changes from stable behavior to oscillatory behavior (cf.Fig 3).

Next to observe the effects of some parameters on system (1), we first consider $c_{3}=0.005$ and observe that the prey population goes to extinction (cf. Fig 4). Next, we consider $\alpha=0.012$ and

Table 1 A set of parameter values.

\begin{tabular}{|l|l|l|}
\hline Parameter & Definition & Default Value \\
\hline$r$ & Growth rate of susceptible prey & 5 \\
\hline$a$ & Growth rate of predator & 0.39 \\
\hline$k$ & Carrying capacity & 80 \\
\hline$\alpha$ & The infectious rate in prey population & 0.07 \\
\hline$k_{1}$ & Half saturation constant in prey population & 0.22 \\
\hline$k_{2}$ & Half saturation constant in predator population & 0.2 \\
\hline$c_{1}$ & Consume rate of susceptible prey by predator & 1 \\
\hline$c_{2}$ & Consume rate of infected prey by predator & 1.2 \\
\hline$c_{3}$ & Maximum value of per-capita reduction rate of predator population & 0.5 \\
\hline$d$ & Mortality rate of infected prey & 0.6 \\
\hline$m$ & Prey refuge rate of infected prey & 0.42 \\
\hline
\end{tabular}

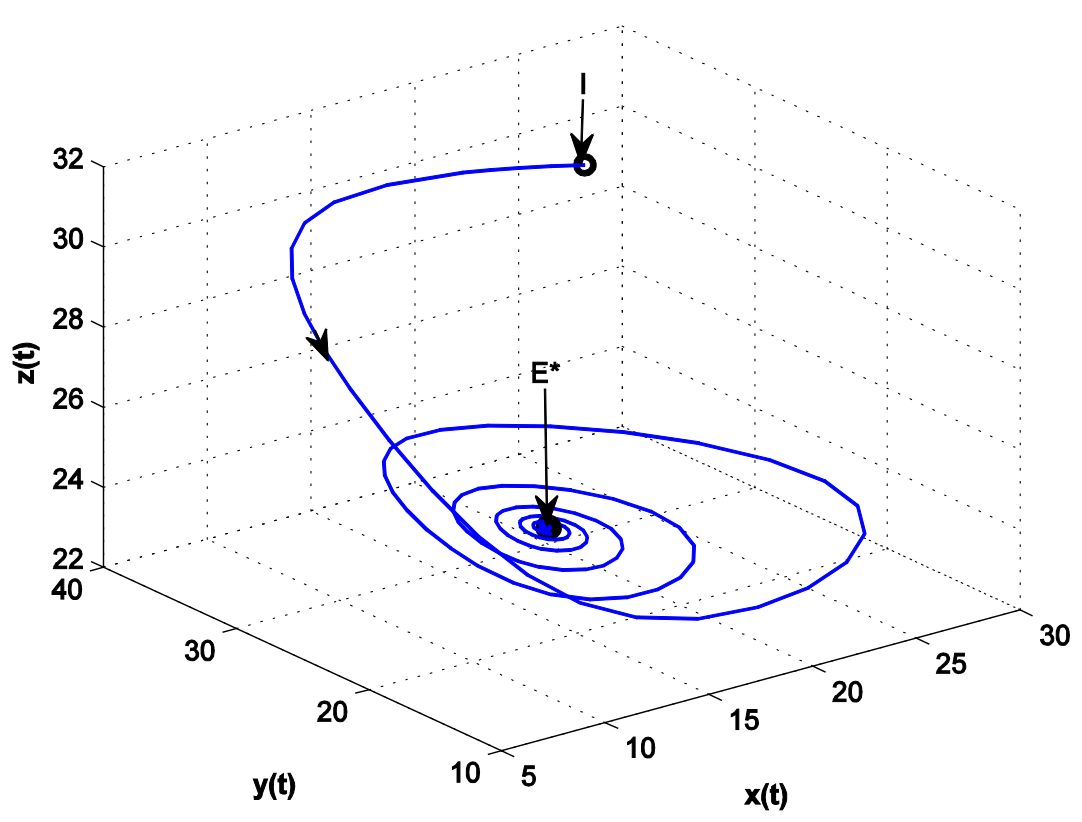

Fig. $1 E^{*}$ is locally asymptotically stable for the set of parameter in the Table 1. 


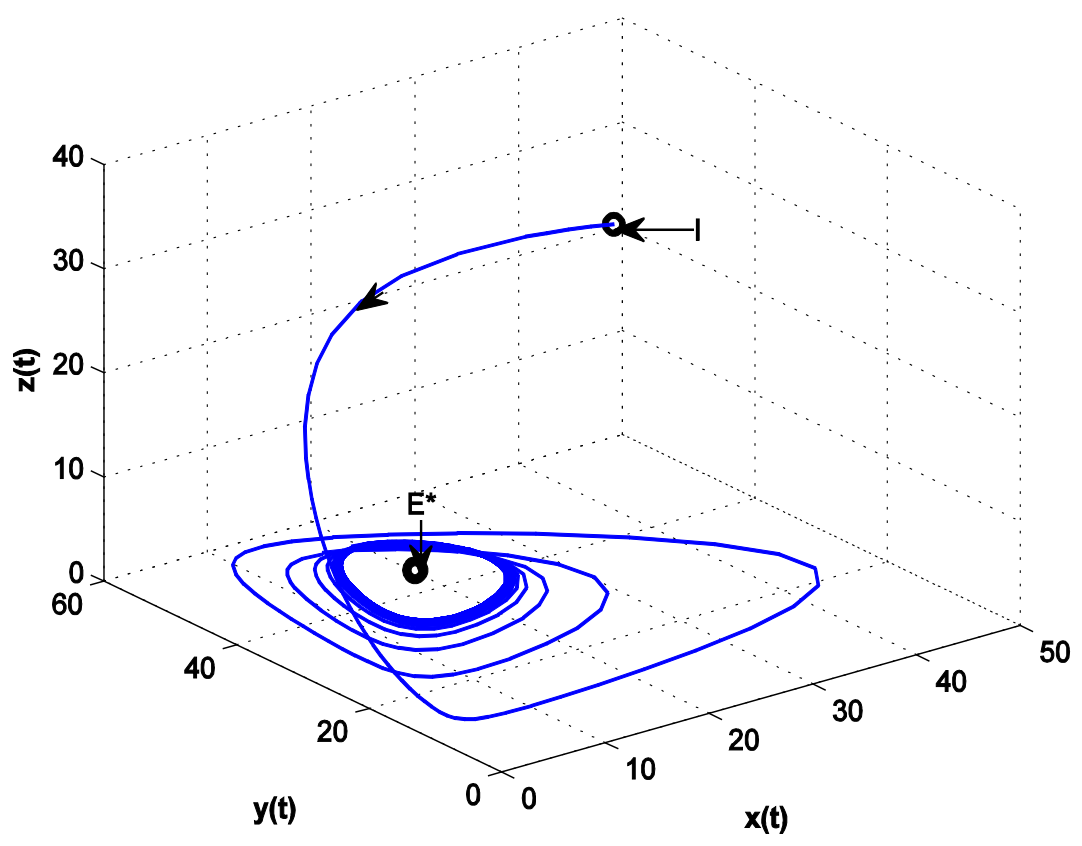

Fig. 2 Oscillatory behaviour of susceptible prey, infected prey and predator for $m=.95$ and other parameters in the Table1.

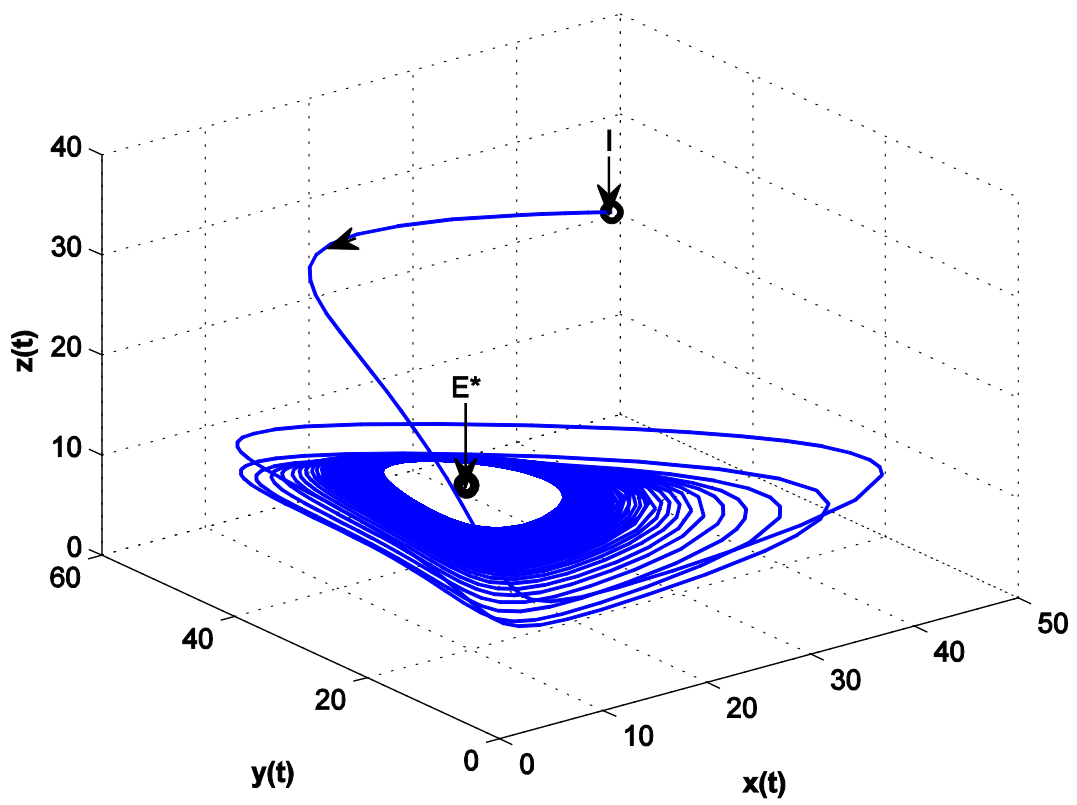

Fig. 3 The figures depicts oscillatory behaviour of susceptible prey, infected prey, predator for $\alpha=.12$ and other parameters in the Table1.

observe that the infected prey population goes to extinction (cf. Fig 5). Finally, for a clear understanding of the dynamical changes of system (1) due to change the value of the parameter $m$, from 0.9 to 1 a bifurcation diagram is plotted as shown in the bifurcation diagram (cf. Fig 6). Also, for a clear understanding of the dynamical changes of system (1) due to change the value of the parameter $\alpha$, from 0.1 to 0.12 a bifurcation diagram is plotted as shown in the bifurcation diagram (cf. Fig 7). 


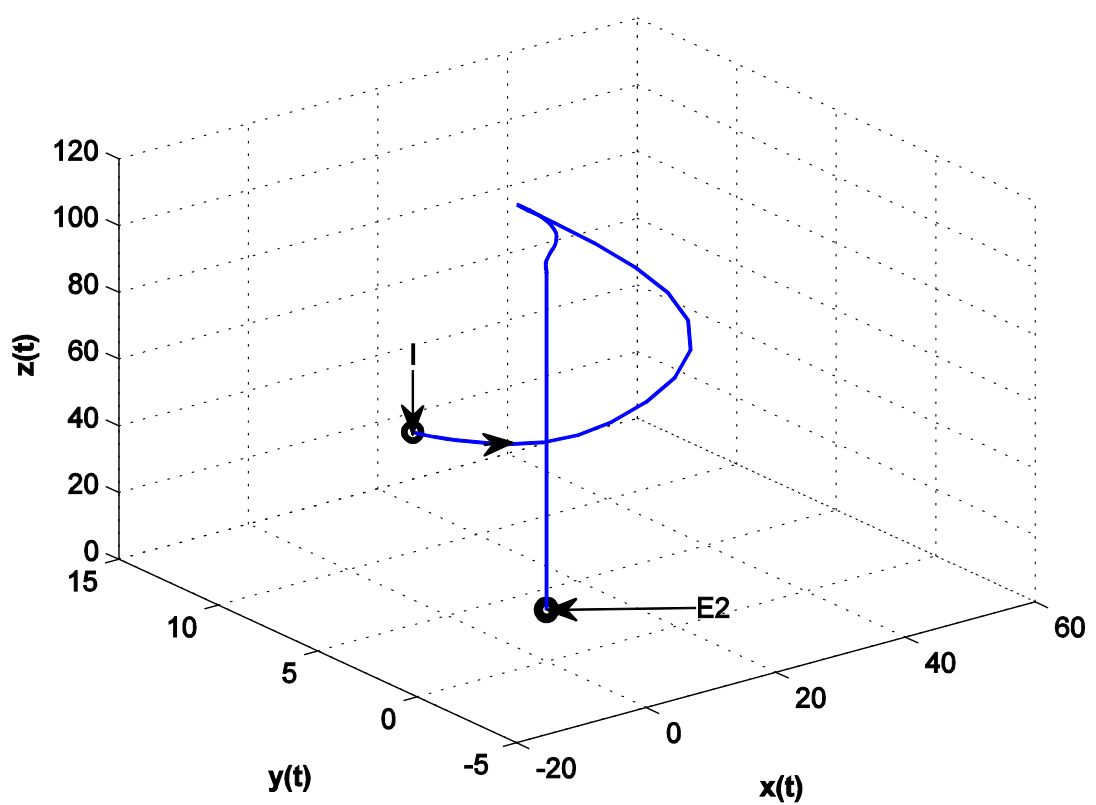

Fig. 4 The figure depicts that for $c_{3}=0.005, E^{*}$ approaches to $E_{2}$ with other parametric values kept fixed in the Table 1.

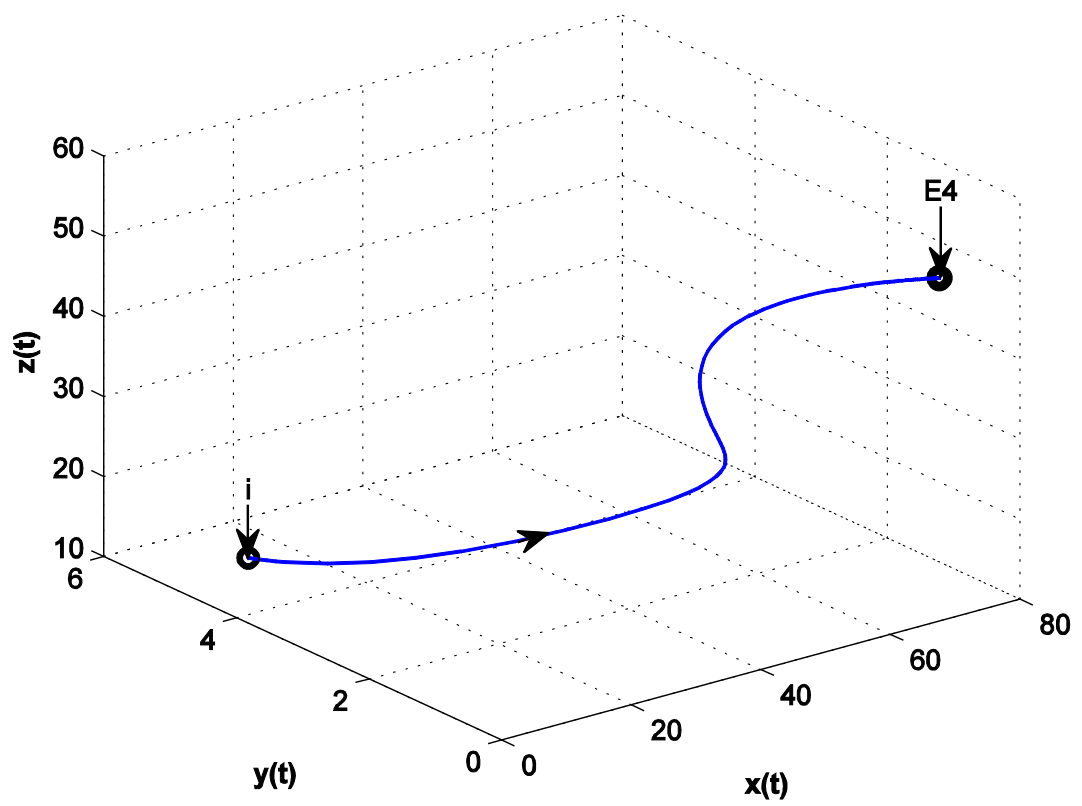

Fig. 5 The figure depicts that for $\alpha=0.012, E^{*}$ approaches to $E_{4}$ with other parametric values kept fixed in the Table 1. 

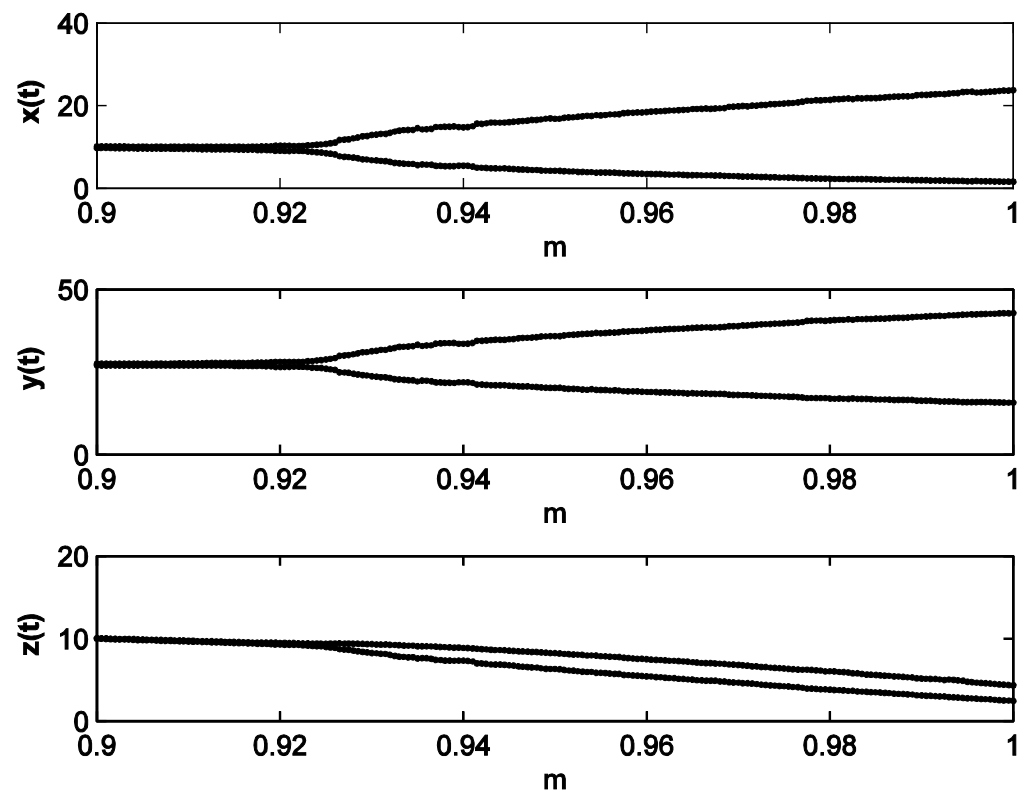

Fig. 6 The bifurcation diagram of all the populations with $m$ as the bifurcation parameter.
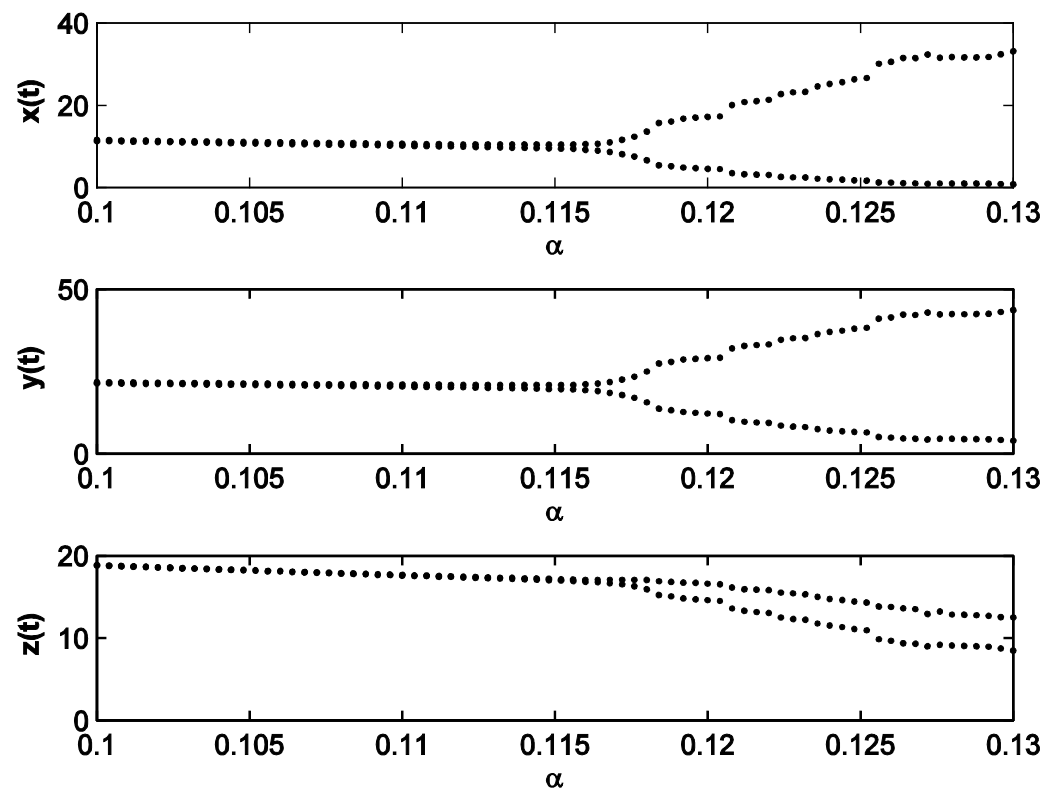

Fig. 7 The bifurcation diagram of all the populations with $\alpha$ as the bifurcation parameter.

\section{Discussion}

In this study we have considered an eco-epidemiological predator-prey mathematical model in which only prey population is infected by disease. As a result the Prey population is divided into two susceptible prey and infected prey. Our model like a modified Leslie-Gower Holling type-II predator-prey model which includes prey refuge as well as infected prey. This model gives us six equilibria, namely one trivial equilibrium $E_{0}$, two axial equilibria $E_{1}, E_{2}$, two planer equilibria $E_{3}, E_{4}$ and one interior equilibrium $E^{*}$. We observe that $E_{0}, E_{1}$ always exists but unstable, $E_{2}$ exists and stable if $R_{01}=\frac{r c_{3} k_{1}}{a c_{1} k_{2}}<1$. But $E_{3}$ exists 
when $R_{1}=\frac{K \alpha}{d}>1$ and it is unstable. Also, $E_{4}$ exists when $R_{2}=\frac{r k_{1} c_{3}}{a c_{1} k_{2}}>1$ and it is stable when $R_{02}=\frac{\alpha c_{3} \bar{x}}{\left(a c_{2}+d c_{3}\right)}<1$. Therefore, if $E_{2}$ is stable then $E_{4}$ does not exists. The interior equilibrium $E^{*}$ exists and it is asymptotically stable under some conditions stated in theorem 2 .

The major difference between the present work and the other recent works as in $[19,32]$ is the incorporation of infected prey refuge leaves a factor of infected prey which are accessible to the predator. Under this additional effect makes the system analyze in this paper more realistic and dynamics than the existing model. There is a great influence of this infected prey refuge on each population has been observed. Both the susceptible prey density and the predator density can be decreased for increasing the amount of infected prey refuge. Whenever the infected prey density is increased, then the interior equilibrium $E^{*}$ of the system (1) becomes stable. Here the prey refuge parameter $(m)$ is an important parameter which acts as stability switch of the system. The stability switching and a Hopf-bifurcation may occur at the interior equilibrium taking prey refuge parameter $(m)$ and rate of infection parameter $(\alpha)$ are bifurcation parameters. We validate all theanalytical results numerically by using Matlab. Finally, it has been observed that our predator-prey mathematical model with infected prey refuge exhibits very interesting dynamics on the basis of the our assumption.

\section{References}

[1] R. M. Anderson and R. M. May, The invasion persistence and spread of infectious diseases within animal and plant communities, Philos Trans $R$ Soc Lond B, vol.314, pp:533-570, 1986.

[2] K.P Hadeler and H.I Freedman, Predator-prey populations with parasitic infection, J Math Biol, vol.27, pp:609-631, 1989.

[3] H.I.Freedman and P. Waltman, Mathematical analysis of some threespecies food chain models, Math Biosci, vol.33, pp:257-276, 1977.

[4] H.I. Freedman and P. Waltman, Persistence in a model of three competitive populations, Math Biosci, vol.73, pp:89-101, 1985.

[5] H.I. Freedman and P. Waltman, Persistence in a model of three interacting predator-prey populations, Math Biosci, vol.68, pp:213-231, 1984.

[6] Y. Takeuchi, Y. Oshime and H. Matsuda, Persistence and periodic orbits of a three-competitor model with refuges, Math Biosci, vol.108, pp:105-125, 1992.

[7] J. T. Tanner, The stability and intrinsic growth rates of prey and predator populations, Ecology, vol.56, pp:855-867, 1975.

[8] P. H. Leslie, A stochastic model for studying the properties of certain biological systems by numerical methods, Biometrika, vol.45, pp:16-31, 1958.

[9] P. H. Leslie, Some further notes on the use of matrices in population mathematics, Biometrika, vol.35, pp:213-245, 1948.

[10] Y. Li and C. Li, Stability and Hopf bifurcation analysis on a delayed Leslie-Gower predator-prey system incorporating a prey refuge, Appl Math Comput, vol.219, pp:4576-4589, 2013.

[11] F. Chen, L. Chen and X. Xie, On a Leslie-Gower predator-prey model incorporating a prey refuge, Nonlinear Anal: Real World Appl, vol. 10, pp:2905-2908, 2009.

[12] Y. Huang, F. Chen and L. Zhong, Stability analysis of a prey-predator model with Holling type II response function incorporating a prey refuge, Appl Math Comput, vol.182, pp:672-683, 2006.

[13] W. Ko and K. Ryu, Qualitative analysis of a predatorprey model with Holling type II functional response incorporating a prey refuge, J Differ Equ, vol.231, pp:534-550, 2006.

[14] H. Chen and J. Sun, Global stability of delay multigroup endemic models with group mixing and nonlinear incidence rates, Appl Math Comput, vol. 218, pp:4391-4400, 2011.

[15] Y. Xue and X. Duan, Dynamic analysis of an SIR epidemic model with nonlinear incidence rate and double delays, Int J Inf Syst Sci, vol.7, pp:92-102, 2011.

[16] S. Wang and Z. Ma, Analysis of an ecoepidemiological model with prey refuges, J Appl Math, 2012. http://dx.doi.org/10.1155/2012/371685.

[17] E. Venturino, The influence of diseases on Lotka-Volterra systems, Rocky Mt J Math, vol.24, pp:381-402, 1994.

[18] E. Venturino, Epidemics in predator-prey models: disease in the predators, IMA J Math Appl Med Biol, vol.19, pp:285-305, 2002.

[19] M. Haque, S. Sarwardi, and E. Venturino,A Leslie-gower 
Holling-type II ecoepidemic model. J App Math Comput, vol.35, pp. 263-280, 2011.

[20] M. Haque, A predatorprey model with disease in the predator species only, Nonlinear Anal Real World Appl, vol.11, pp:2224-2236, 2010.

[21] M. Haque and D. Greenhalgh, When Predator avoids infected prey: a model based theoretical studies, IMA J Math Med Biol, vol.27, pp:75-94, 2009.

[22] M. Haque, J. Zhen and E. Venturino, Rich dynamics of Lotka-Volterra type predator-prey model system with viral disease in Prey species, Math Methods Appl Sci, vol.32, pp: 875-898, 2009.

[23] Y. N. Xiao and L.S. Chen, Analysis of a three species eco-epidemiological model, $J$ Math Anal Appl, vol.258(2), pp:733-754, 2001.

[24] Y. N. Xiao and L.S. Chen, Modelling and analysis of a predator-prey model with disease in the prey, Math Biosci, vol.171, pp:59-82, 2001.

[25] J. J. Tewa, V. Y. Djeumen and S. Bowong, Predator-prey model with Holling response function of type II and SIS infectious disease, Appl Math Model, vol.37, pp:4825-4841, 2013.
[26] H. Hethcote, W. Wang, L. Han L and Z. Ma, A predator-prey model with infected prey, Theor Popul Biol, vol.66, pp:259-268, 2004.

[27] Md. S. Rahman and S. Chakravarty, A predator-prey model with disease in prey, Nonlinear Anal: Model Cont, vol.18, pp:191-209, 2013.

[28] K. Das, K. Kundu, J. Chattopadhyay, "A predatorprey mathematical model with both the populations affected by diseases,"Ecol. Complex. vol. 8, pp. 68-80, 2011.

[29] J. Chattopadhyay, S. Pal, A.E. Abdllaoui, "Classical predatorprey system with infection of prey population mathematical model," Math. Methods Appl.Sci., vol. 26, no. 14, pp. 1211-1222, 2003.

[30] J. Chattopadhyay, O. Arino, "A predatorprey model with disease in the prey,"Nonlinear Anal., vol. 36, pp. 747-766, 1999.

[31] Birkhoff, G., Rota, G. C. "Ordinary Differential equations." it Ginn Boston, 1982.

[32] M. Haque, and E. Venturino, The role of transmissible disease in the Holling-Tanner predator-prey model. Theoretical Population Biology, vol.70, pp. 273-28, 2011. 


\section{A Leslie-Gower Holling Type-II Predator-Prey Mathematical Model with Disease in \\ Prey Population Incorporating a Prey Refuge}

Appendix

Trivial equilibrium $E_{0}$

The variational matrix of the system $(1)$ at $E_{0}(0,0,0)$ is given by

$$
V\left(E_{0}\right)=\left[\begin{array}{ccc}
r & 0 & 0 \\
0 & -d & 0 \\
0 & 0 & a
\end{array}\right]
$$

So, the eigenvalues of the characteristic equation of $V\left(E_{0}\right)$ are $\mathrm{r},-\mathrm{d}$ and a. Since two eigenvalue are positive, the equilibrium point $E_{0}$ is always unstable for all parametric values.

Axial equilibria $E_{1}$ and $E_{2}$

(i) The variational matrix of the system (1) at $E_{1}(k, 0,0)$ is given by

$$
V\left(E_{1}\right)=\left[\begin{array}{ccc}
-r & -(r+k \alpha) & -\frac{c_{1} k}{\left(k+k_{1}\right)} \\
0 & \alpha k-d & 0 \\
0 & 0 & a
\end{array}\right]
$$

So, the eigenvalues of the characteristic equation of $V\left(E_{1}\right)$ are -r, $\alpha k-d$ and a. Since one eigenvalues is positive, the equilibrium point $E_{1}$ is always unstable for all parametric values.

(ii) The variational matrix of the system (1) at $E_{2}\left(0,0, \frac{k_{2} a}{c_{3}}\right)$ is given by

$$
V\left(E_{2}\right)=\left[\begin{array}{ccc}
-\left(\frac{a c_{1} k_{2}}{k_{1} c_{3}}-r\right) & 0 & 0 \\
0 & -\left(\frac{a c_{2}}{c_{3}}+d\right) & 0 \\
\frac{a^{2}}{c_{3}} & \frac{a^{2}(1-m)}{c_{3}} & -a
\end{array}\right]
$$

Therefore, the eigenvalues of the characteristic equation of $V_{2}$ are $-\frac{a c_{1} k_{2}}{c_{3} k_{1}}\left(1-\frac{c_{3} k_{1} r}{c_{1} k_{2} a}\right),-\left(\frac{a c_{2}}{c_{3}}+d\right)$ and $-\mathrm{a}$. So $E_{2}$ is stable if $R_{01}<1$,where $R_{01}=\frac{c_{3} k_{1} r}{c_{1} k_{2} a}$.

Planer equilibria $E_{3}$ and $E_{4}$

(i) The variational matrix of the system (1) around the equilibrium point $E_{3}\left(\frac{d}{\alpha}, \frac{r(k \alpha-d)}{\alpha(k \alpha+r)}, 0\right)$ is 


$$
V\left(E_{3}\right)=\left[\begin{array}{ccc}
-\left(\frac{r d}{k \alpha}-r\right) & -\frac{d(k \alpha+r)}{\alpha k} & -\frac{c_{1} d(k \alpha+r)}{\left(\alpha k_{1}+d\right)(k \alpha+r)+r \alpha(k \alpha-r)(1-m)} \\
\frac{r(k \alpha-d)}{k \alpha+r} & 0 & -\frac{r c_{2}(k \alpha-d)(1-m)}{\left(\alpha k_{2}+d\right)(k \alpha+r)+r(k \alpha-d)(1-m)} \\
0 & 0 & a
\end{array}\right]
$$

The characteristic roots of the variational matrix computed around $E_{3}$ are $a(>0)$ and the roots of the equation

$$
\rho^{2}+\frac{r d}{k \alpha} \rho+\frac{r d(k \alpha-d)}{\alpha k}=0
$$

Since one of the eigenvalues is the equilibrium point $E_{3}$ is positive i.e., $a(>0)$ so the equilibrium point $E_{3}$ is unstable for all parametric values.

(ii) The variational matrix of the system (1) around the equilibrium point $\mathbf{E}_{4}(\bar{x}, 0, \bar{z})$ is

$$
V\left(E_{4}\right)=\left[\begin{array}{lll}
m_{11} & m_{12} & m_{13} \\
m_{21} & m_{22} & m_{23} \\
m_{31} & m_{32} & m_{33}
\end{array}\right]
$$

where

$$
\begin{gathered}
m_{11}=r-\frac{2 r}{k} \bar{x}-\frac{c_{1} k_{1} \bar{z}}{\left(x+k_{1}\right)^{2}}, \quad m_{12}=-\left(\frac{r}{k}+\alpha\right) \bar{x}+\frac{c_{1}(1-m) \overline{x z}}{\left(x+k_{1}\right)^{2}}, m_{13}=-\frac{c_{1} \bar{x}}{x+k_{1}} \\
m_{21}=0, \quad m_{22}=\alpha \bar{x}-\frac{c_{2} \bar{z}}{x+k_{2}}-d=\alpha \bar{x}-\frac{a c_{2}}{c_{3}}-d, \quad m_{23}=0 \\
m_{31}=\frac{a^{2}}{c_{3}}, \quad m_{32}=\frac{a^{2}(1-m)}{c_{3}}, \quad m_{33}=-a ;
\end{gathered}
$$

It is found that the characteristic roots of the variational matrix $V_{3}$ at $E_{3}$ are

$$
\begin{gathered}
-\frac{\left(a c_{2}+d c_{3}\right)}{c_{3}}\left(1-\frac{c_{3} \alpha \bar{x}}{\left(a c_{2}+d c_{3}\right)}\right) \text { and the roots of the equation } \\
w^{2}+Q_{1} w+Q_{2}=0
\end{gathered}
$$

where $Q_{1}=a+\frac{2 r \bar{x}}{k}+\frac{c_{1} k_{1} \bar{z}}{\left(x+k_{1}\right)^{2}}-r>0 ; Q_{2}=a\left\{\frac{2 r}{k} \bar{x}+\frac{c_{1} \bar{z}}{\left(x+k_{1}\right)^{2}}+\frac{a c_{1} \bar{x}}{c_{3}\left(x+k_{1}\right)}-r\right\}>0$. Thus $E_{3}$ is stable if $R_{02}<1$, where $R_{02}=\frac{c_{3} \alpha \bar{x}}{\left(a c_{2}+d c_{3}\right)}$, so that $E_{3}$ is stable. 